\title{
A Fixed Point Theorem for Monotone Maps and Its Applications to Nonlinear Matrix Equations
}

\author{
Dongjie Gao \\ Department of Mathematics, Heze University, Heze, Shandong 274015, China \\ Correspondence should be addressed to Dongjie Gao; aizai_2004@126.com
}

Received 25 July 2015; Revised 27 October 2015; Accepted 23 November 2015

Academic Editor: Frank Uhlig

Copyright (C) 2015 Dongjie Gao. This is an open access article distributed under the Creative Commons Attribution License, which permits unrestricted use, distribution, and reproduction in any medium, provided the original work is properly cited.

By using the fixed point theorem for monotone maps in a normal cone, we prove a uniqueness theorem for the positive definite solution of the matrix equation $X=Q+A^{*} f(X) A$, where $f$ is a monotone map on the set of positive definite matrices. Then we apply the uniqueness theorem to a special equation $X=k Q+A^{*}(\widehat{X}-C)^{q} A$ and prove that the equation has a unique positive definite solution when $\widehat{Q} \geq C$ and $k>1$ and $0<q<1$. For this equation the basic fixed point iteration is discussed. Numerical examples show that the iterative method is feasible and effective.

\section{Introduction}

We consider the matrix equation

$$
X=Q+A^{*} f(X) A,
$$

where $Q$ is an $n \times n$ positive definite matrix, $A$ is arbitrary $m \times$ $n$ matrix, and $f$ is a monotone map on $P(m)$.

The study of matrix equation has a long history, involving in particular the study of algebraic Riccati equations for discrete time optimal control and for the stochastic realization problem. Motivated by these equations, somewhat simpler versions, namely, (1) with $f(X)= \pm X^{-1}$, were studied in [1-3]. Those three papers were the start of a development. Later on came papers on a number of other specific matrix equations, such as papers [4-10].

Of particular interest is the equation $X=Q+A^{*}(\widehat{X}-$ $C)^{-1} A$, where $\widehat{X}$ is the Kronecker product of $I_{p}$ with $X$ for some $p$. This equation is connected to an interpolation problem proposed by Sakhnovich in [11]. This equation was first studied in [12], and a perturbation analysis was discussed in [13]. Recently, in [14], the author provided a new proof for the uniqueness of the positive definite solution of this equation using a change of variable and a fixed point theorem, which is an easier argument than the one used in [12].

This development leads to consideration of a general class of matrix equations, which started with the paper by El-Sayed and Ran [15], and was developed further by Ran and Reurings [16-19].

In this paper, we are interested in positive definite solutions of (1), where $f$ is a monotone map. We obtain a uniqueness theorem by using the fixed point theorem for monotone maps in a normal cone. The uniqueness theorem can be widely used in nonlinear matrix equations involving monotonicity. In addition, we apply the uniqueness theorem to a special equation and discuss the basic fixed point iteration for this special case.

The following notations are used throughout this paper. Let $H(n)$ denote $n \times n$ Hermitian matrices, let $\mathscr{M}(m, n)$ denote $m \times n$ matrices, let $\mathscr{P}(n)$ denote $n \times n$ positive definite matrices, and let $\overline{\mathscr{P}}(n)$ denote $n \times n$ positive semidefinite matrices. For $X, Y \in \mathscr{P}(n)$, we write $X \geq Y(X>Y)$ if $X-Y$ is positive semidefinite (definite). $A^{*}$ denotes the conjugate transpose of a matrix $A$. Let $P$ denote a solid cone of a real Banach space E. $P^{0}$ denotes the interior points set of $P$. A cone is said to be a solid cone if $P^{0} \neq \phi$.

\section{Preliminaries}

In this section, we introduce some definitions and properties for monotone operators in a normal cone which are the theoretical basis of this paper. 
Definition 1 (see [20]). A cone $P \subset E$ is said to be normal if there exists a constant $N>0$ such that $0 \leq x \leq y$ implies $\|x\| \leq N\|y\|$. That is, the norm $\|\cdot\|$ is semimonotone.

Definition 2 (see [20]). The operator $\Gamma: D \rightarrow E, D \subset E$, is said to be an increasing operator if

$$
x_{1} \geq x_{2}, \quad x_{1}, x_{2} \in D \Longrightarrow \Gamma\left(x_{1}\right) \geq \Gamma\left(x_{2}\right) .
$$

$\Gamma$ is said to be an decreasing operator if

$$
x_{1} \geq x_{2}, \quad x_{1}, x_{2} \in D \Longrightarrow \Gamma\left(x_{1}\right) \leq \Gamma\left(x_{2}\right) \text {. }
$$

Definition 3 (see [21]). Let $P$ be a solid cone of a real Banach space $E$ and $\Gamma: P^{0} \rightarrow P^{0}$. Let $0 \leq \alpha<1$. Then $\Gamma$ is said to be $\alpha$-concave if

$$
\Gamma(t x) \geq t^{\alpha} \Gamma(x) \quad \forall x \in P^{0}, 0<t<1 .
$$

$\Gamma$ is said to be $(-\alpha)$-convex if

$$
\Gamma(t x) \leq t^{-\alpha} \Gamma(x) \quad \forall x \in P^{0}, 0<t<1 .
$$

Lemma 4 (see [21]). Let $P$ be a normal cone of a real Banach space $E$ and let $\Gamma: P^{0} \rightarrow P^{0}$ be $\alpha$-concave and increasing (or $(-\alpha)$-convex and decreasing) for $0 \leq \alpha<1$. Then $\Gamma$ has exactly one fixed point $x$ in $P^{0}$.

Lemma 5 (see [20]). A cone $P$ is normal if and only if $x_{n} \leq$ $z_{n} \leq y_{n}, x_{n} \rightarrow x$, and $y_{n} \rightarrow x$ imply $z_{n} \rightarrow x$.

Lemma 6 (see [22]). For all $A \in H(n)$ and $r \in[0,1]$, the operator $\Phi$ given by $\Phi(A)=A^{r}$ is an increasing operator. Then $A^{r} \geq B^{r}$ if $A, B \in H(n), A \geq B$, and $0 \leq r \leq 1$.

In the following, we will apply Lemma 4 to the map $G(X)=Q+A^{*} f(X) A$.

\section{The Application to $G(X)=Q+A^{*} f(X) A$}

We define the spectral norm $\|\cdot\|$ in $H(n)$; then $H(n)$ is a real Banach space. It is well known that $\bar{P}(n)$ is a cone in $H(n)$ and the interior points set is $P(n)$. Since the spectral norm is monotone, we have from Definition 1 that the set $\bar{P}(n)$ is normal cone. So we can apply the results in Section 2 to the maps from $P(n)$ into $P(n)$. In the following, we will consider the positive definite solutions of the equation

$$
X=Q+A^{*} f(X) A
$$

or equivalently the fixed points of the map

$$
G(X)=Q+A^{*} f(X) A,
$$

where $f$ is a monotone map on $P(m)$ induced by a real valued map on $(0, \infty)$. The following theorem is our main result in this section.

Theorem 7. Let $G(X)=Q+A^{*} f(X) A, Q \in P(n), A \in$ $M(m, n)$. Then $G$ has exactly one fixed point $X$ in $P(n)$ if

(1) $f: \bar{P}(m) \rightarrow \bar{P}(m)$;
(2) $f$ is increasing and $\alpha$-concave or decreasing and $(-\alpha)$ convex.

Proof. For the application of Lemma 4, we set $E=H(n), P=$ $\bar{P}(n), P^{0}=P(n)$, and $\Gamma=G$. Now we will prove that the map $G$ satisfies the conditions of Lemma 4 .

For all $X, Y \in P(n)$ with $X \geq Y$, by Lemma 6 , we have the following:

(1) $G: P(n) \rightarrow P(n)$, because $f$ maps $\bar{P}(m)$ into itself and $Q \in P(n)$.

(2) If $f$ is increasing and $\alpha$-concave, then $G$ is increasing. For all $t \in(0,1)$, we have

$$
\begin{aligned}
G(t X) & =Q+A^{*} f(t X) A \geq t^{\alpha} Q+t^{\alpha} A^{*} f(X) A \\
& =t^{\alpha}\left[Q+A^{*} f(X) A\right]=t^{\alpha} G(X) .
\end{aligned}
$$

Hence the map $G$ is increasing and $\alpha$-concave.

If $f$ is decreasing and $(-\alpha)$-convex, then $G$ is decreasing. For all $t \in(0,1)$, we have

$$
\begin{aligned}
G(t X) & =Q+A^{*} f(t X) A \leq t^{-\alpha} Q+t^{-\alpha} A^{*} f(X) A \\
& =t^{-\alpha}\left[Q+A^{*} f(X) A\right]=t^{-\alpha} G(X) .
\end{aligned}
$$

Hence the map $G$ is decreasing and $(-\alpha)$-concave.

So the map $G$ satisfies all the conditions in Lemma 4. According to Lemma 4, $G$ has exactly one fixed point $X$ in $P(n)$.

The conditions in this theorem which $f$ has to satisfy are easy to check if $f$ is simple. Now, we will give two simple examples.

Example 8. If $f(X)=X^{q}, 0<q<1$, then $G(X)=Q+A^{*} X^{q} A$ has exactly one fixed point $X$ in $P(n)$.

Proof. By Lemma $6 f: \bar{P}(n) \rightarrow \bar{P}(n)$ is increasing. Also

$$
f(t X)=(t X)^{q}=t^{q} X^{q}=t^{q} f(X) .
$$

Let $\alpha=q$; then $f$ is $\alpha$-concave. According to Theorem 7, $G(X)=Q+A^{*} X^{q} A$ has exactly one fixed point $X$ in $P(n)$.

Example 9. If $f(X)=X^{-q}, 0<q<1$, then $G(X)=Q+$ $A^{*} X^{-q} A$ has exactly one fixed point $X$ in $P(n)$.

Proof. By Lemma $6 f: \bar{P}(n) \rightarrow \bar{P}(n)$ is decreasing. Also

$$
f(t X)=(t X)^{-q}=t^{-q} X^{-q}=t^{-q} f(X) .
$$

Let $\alpha=q$; then $f$ is $(-\alpha)$-convex. According to Theorem 7, $G(X)=Q+A^{*} X^{-q} A$ has exactly one fixed point $X$ in $P(n)$.

These two examples have been discussed in several papers by other methods; see, for example, $[7,8]$. It seems that the argument presented here is simpler than the arguments of [7, $8]$. 


\section{The Case of $G(X)=k Q+A^{*}(\widehat{X}-C)^{q} A$}

In this section, we will discuss a more complex map; namely, $G(X)=k Q+A^{*}(\widehat{X}-C)^{q} A$. Here $\widehat{X}$ is the block diagonal matrix defined by $\widehat{X}=\operatorname{diag}(X, X, \ldots, X)$, in which $X$ is an $n \times n$ matrix. Also, $Q$ is an $n \times n$ positive definite matrix, $C$ is an $m n \times m n$ positive semidefinite matrix, and $A$ is arbitrary $m n \times n$ matrix. we always assume that $\widehat{Q} \geq C, k>1$, and $0<q<1$. under these conditions we discuss the positive definite solutions of the equation

$$
X=k Q+A^{*}(\widehat{X}-C)^{q} A
$$

or equivalently the fixed points of the map $G(X)$. In this case, $f(X)=(\widehat{X}-C)^{q}$. The function $f$ is increasing, but it seems to be hard to prove that $f$ is $\alpha$-concave. Therefore, we will use a change of variable to study an equivalent form of the map G.

Let $S_{1}(n) \subset P(n)$ be the set defined by

$$
S_{1}(n)=\{X \in P(n) \mid X>Q\} .
$$

Let $C_{1}=\widehat{Q}-C$. For $\widehat{Q} \geq C$, we know that $C_{1}$ is a positive semidefinite matrix. Then (12) turns into

$$
X-Q=(k-1) Q+A^{*}\left(\widehat{X}-\widehat{Q}+C_{1}\right)^{q} A .
$$

Let $Y=X-Q$. Then (12) eventually becomes

$$
Y=(k-1) Q+A^{*}\left(\widehat{Y}+C_{1}\right)^{q} A .
$$

Apparently, (12) is equivalent to (15) when $X=Y+Q$. Thus, we can obtain the following conclusion.

Lemma 10. Suppose that $\widehat{Q} \geq C, k>1$; then $X$ is a positive definite solution of (12) if and only if $Y=X-Q$ is a positive definite solution of (15).

Theorem 11. Equation (12) with $\widehat{Q} \geq C$ and $k>1$ always has a unique positive definite solution.

Proof. According to Lemma 10, we first consider (15). Define $G_{1}$ by $G_{1}(Y)=(k-1) Q+A^{*}\left(\widehat{Y}+C_{1}\right)^{q} A$ and $f_{1}(Y)=$ $\left(\widehat{Y}+C_{1}\right)^{q}$. Now we will prove that the operator $G_{1}$ satisfies the conditions of Theorem 7 .

For all $X, Y \in P(n)$ with $X \geq Y$, by Lemma 6 , we have the following:

(1) $G_{1}: P(n) \rightarrow P(n)$;

(2) $f_{1}(X)=\left(\widehat{X}+C_{1}\right)^{q} \geq\left(\widehat{Y}+C_{1}\right)^{q}=f_{1}(Y)$;

(3) for all $t \in(0,1)$, we have

$$
\begin{aligned}
f_{1}(t Y) & =\left(\widehat{t Y}+C_{1}\right)^{q}=t^{q}\left(\widehat{Y}+t^{-1} C_{1}\right)^{q} \\
& \geq t^{q}\left(\widehat{Y}+C_{1}\right)^{q}=t^{q} f_{1}(Y) .
\end{aligned}
$$

Hence, the operator $f_{1}$ is increasing and $\alpha$-concave. From Theorem 7 we get that the operator $G_{1}$ has a unique fixed point $Y$ in $P(n)$, which is the unique positive definite solution of (15). According to Lemma 10, (12) has a unique positive definite solution.
Now we consider the following iterative method for (15) and (12). Let

$$
\begin{aligned}
& Y_{n+1}=(k-1) Q+A^{*}\left(\widehat{Y}_{n}+C_{1}\right)^{q} A, \\
& n=0,1,2, \ldots, \\
& X_{n+1}=k Q+A^{*}\left(\widehat{X}_{n}-C\right)^{q} A, \quad n=0,1,2, \ldots .
\end{aligned}
$$

For the matrix sequence $\left\{X_{n}\right\}$ defined by (18), we have the following theorem.

Theorem 12. Suppose that $\widehat{Q} \geq C, k>1$; then for arbitrary initial matrix $X_{0} \in S_{1}(n)$, the matrix sequence $\left\{X_{n}\right\}$ defined by (18) converges to the unique positive definite solution $X$ of (12).

Proof. We first consider the matrix sequence $\left\{Y_{n}\right\}$ defined by (17). Let $Y=X-Q, Y_{0}=X_{0}-Q$; then $Y$ is the unique positive definite solution of (15). For $Y$ and $Y_{0}$, there exists a positive number $0<\alpha \leq 1$ satisfying

$$
\alpha Y \leq Y_{0} \leq \alpha^{-1} Y .
$$

We will use mathematical induction to prove the following inequality:

$$
\alpha^{q^{n}} Y \leq Y_{n} \leq\left(\alpha^{-1}\right)^{q^{n}} Y, \quad n=0,1,2, \ldots
$$

From (19) it follows that inequality (20) holds for $n=0$. Assume that (20) is true for $n=k$. That is,

$$
\alpha^{q^{k}} Y \leq Y_{k} \leq\left(\alpha^{-1}\right)^{q^{k}} Y .
$$

Now we need to prove (20) is true for $n=k+1$. From (21), we get that

$$
\begin{aligned}
& \alpha^{q^{k}} \widehat{Y}+C_{1} \leq \widehat{Y_{k}}+C_{1} \leq\left(\alpha^{-1}\right)^{q^{k}} \widehat{Y_{k}}+C_{1}, \\
& \left(\alpha^{q^{k}} \widehat{Y}+C_{1}\right)^{q} \leq\left(\widehat{Y_{k}}+C_{1}\right)^{q} \leq\left[\left(\alpha^{-1}\right)^{q^{k}} \widehat{Y}+C_{1}\right]^{q}, \\
& (k-1) Q+A^{*}\left(\alpha^{q^{k}} \widehat{Y}+C_{1}\right)^{q} A \\
& \leq(k-1) Q+A^{*}\left(\widehat{Y_{k}}+C_{1}\right)^{q} A \\
& \leq(k-1) Q+A^{*}\left[\left(\alpha^{-1}\right)^{q^{k}} \widehat{Y}+C_{1}\right]^{q} A .
\end{aligned}
$$

That is,

$$
\begin{aligned}
& (k-1) Q+A^{*}\left(\alpha^{\alpha^{k}} \widehat{Y}+C_{1}\right)^{q} A \leq Y_{k+1} \\
& \leq(k-1) Q+A^{*}\left[\left(\alpha^{-1}\right)^{q^{k}} \widehat{Y}+C_{1}\right]^{q} A .
\end{aligned}
$$


TABLE 1

\begin{tabular}{lcccc}
\hline errtol & $q$ & $\operatorname{err}(X)$ & $n$ & Iteration solution $X$ \\
\hline $1 \times 10^{-5}$ & $1 / 3$ & $9.4202 \times 10^{-6}$ & 11 & $\left(\begin{array}{ccc}2.13388783159709 & -0.00086471161567 \\
-0.00086471161567 & 0.53349253880560\end{array}\right) \times 10^{3}$ \\
& $1 / 2$ & $5.2724 \times 10^{-6}$ & 19 & -0.13190912090591 \\
$1 \times 10^{-10}$ & $1 / 3$ & $3.7231 \times 10^{-11}$ & 22 & $\left(\begin{array}{ccc}2.28733182761235 & \\
-0.13190912090591 & 0.47447253811381\end{array}\right) \times 10^{4}$ \\
\hline & $1 / 2$ & $7.6719 \times 10^{-11}$ & 35 & $\left(\begin{array}{ccc}2.13391707124142 & -0.00086517759943 \\
-0.00086517759943 & 0.53349925037736\end{array}\right) \times 10^{3}$ \\
& & & $\left(\begin{array}{ccc}2.28735583246287 & -0.13191055029808 \\
-0.13191055029808 & 0.47447745556329\end{array}\right) \times 10^{4}$ \\
\hline
\end{tabular}

From $0<\alpha \leq 1$ it follows that $0<\alpha^{q^{k+1}} \leq 1,\left(\alpha^{-1}\right)^{q^{k+1}} \geq 1$. Then

$$
\begin{aligned}
&(k-1) Q+A^{*}\left(\alpha^{q^{k}} \widehat{Y}+C_{1}\right)^{q} A \\
& \geq \alpha^{q^{k+1}}(k-1) Q+A^{*}\left(\alpha^{q^{k}} \widehat{Y}+\alpha^{q^{k}} C_{1}\right)^{q} A \\
&= \alpha^{q^{k+1}}\left[(k-1) Q+A^{*}\left(\widehat{Y}+C_{1}\right)^{q} A\right], \\
&(k-1) Q+A^{*}\left[\left(\alpha^{-1}\right)^{q^{k}} \widehat{Y}+C_{1}\right]^{q} A \\
& \leq\left(\alpha^{-1}\right)^{q^{k+1}}(k-1) Q \\
& \quad+A^{*}\left[\left(\alpha^{-1}\right)^{q^{k}} \widehat{Y}+\left(\alpha^{-1}\right)^{q^{k}} C_{1}\right]^{q} A \\
&=\left(\alpha^{-1}\right)^{q^{k+1}}\left[(k-1) Q+A^{*}\left(\widehat{Y}+C_{1}\right)^{q} A\right] .
\end{aligned}
$$

Therefore

$$
\begin{aligned}
& \alpha^{q^{k+1}}\left[(k-1) Q+A^{*}\left(\widehat{Y}+C_{1}\right)^{q} A\right] \leq Y_{k+1} \\
& \quad \leq\left(\alpha^{-1}\right)^{q^{k+1}}\left[(k-1) Q+A^{*}\left(\widehat{Y}+C_{1}\right)^{q} A\right] .
\end{aligned}
$$

That is,

$$
\alpha^{q^{k+1}} Y \leq Y_{k+1} \leq\left(\alpha^{-1}\right)^{q^{k+1}} Y
$$

Hence we get that inequality (20) holds for any positive integer $n$. Let $n \rightarrow \infty$; we have

$$
\alpha^{q^{n}} \longrightarrow 1, \quad\left(\alpha^{-1}\right)^{q^{n}} \longrightarrow 1 .
$$

Therefore, from Lemma 5 it follows that

$$
Y_{n} \longrightarrow Y \text {. }
$$

Since $X_{n}=Y_{n}+Q, X=Y+Q$, we have

$$
X_{n} \longrightarrow X
$$

\section{Numerical Examples}

We now present some numerical examples to illustrate our results. All computations were performed using MATLAB, version 7.01. In this section, we will use $\operatorname{err}(X)=\| X-k Q-$ $A^{*}(\widehat{X}-C)^{q} A\left\|_{1} /\right\| X \|_{1}$ to denote the relative iteration error, errtol to denote the stopping criterion, and $n$ to denote the iteration number.

Example 13. Consider (12) with $k=2$ and

$$
\begin{aligned}
& A=\left(\begin{array}{cc}
3 & -2 \\
2 & 0 \\
-8 & 3 \\
7 & 2 \\
-5 & 1 \\
7 & 6
\end{array}\right) \\
& Q=\left(\begin{array}{rr}
23 & -2 \\
-2 & 15
\end{array}\right) \text {, } \\
& C=\left(\begin{array}{cccccc}
9 & -1 & 2 & 0 & 4 & 1 \\
-1 & 8 & -1 & -1 & 3 & -2 \\
2 & -1 & 6 & 1 & -1 & 2 \\
0 & -1 & 1 & 7 & 0 & -2 \\
4 & 3 & -1 & 0 & 8 & 0 \\
1 & -2 & 2 & -2 & 0 & 5
\end{array}\right) .
\end{aligned}
$$

Then the matrices $Q$ and $C$ satisfy $\widehat{Q}>C$. Consider the iterative method (18) with several values of $q$ and several values of the stopping criterion. The experiment data are listed in Table 1.

MATLAB function $m$-file is shown in Algorithm 1. 


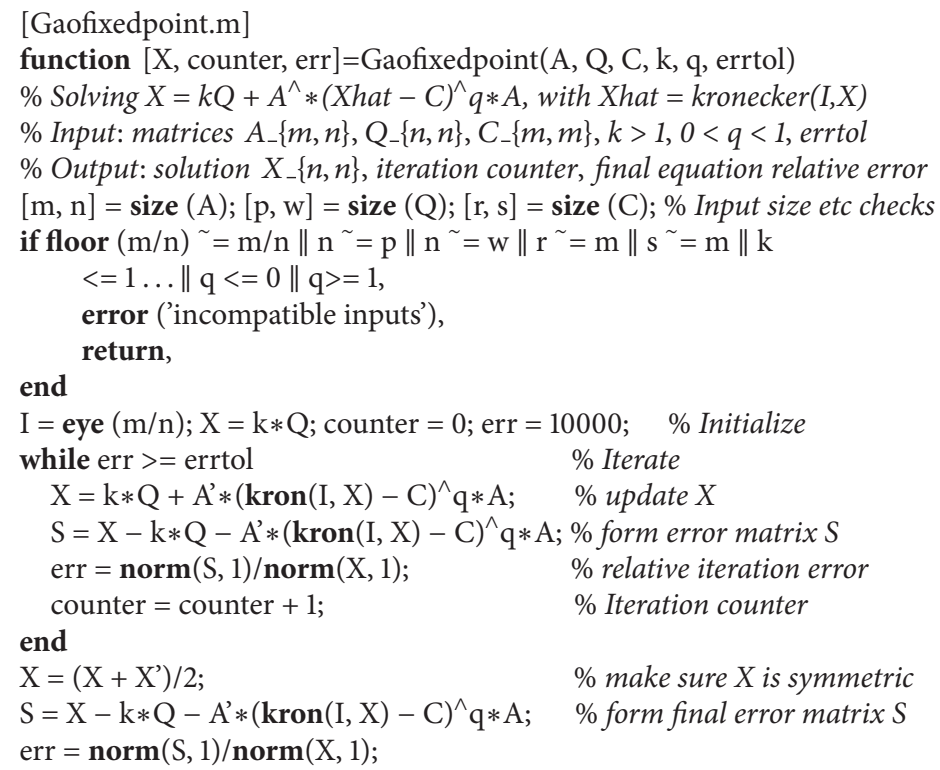

Algorithm 1

\section{Conflict of Interests}

The author declares that there is no conflict of interests regarding the publication of this paper.

\section{Acknowledgments}

The author wishes to thank Professor Frank Uhlig and the anonymous referees for providing very useful suggestions for improving this paper. The work was supported by the National Natural Science Foundation of China (11071141), the Natural Science Foundation of Shandong Province of China (ZR2015AL017ZR2014AM032), and the Project of Shandong Province Higher Educational Science and Technology Program (J11LA06, J13LI02).

\section{References}

[1] J. C. Engwerda, "On the existence of a positive definite solution of the matrix equation $X+A^{T} X^{-1} A=I$," Linear Algebra and Its Applications, vol. 194, pp. 91-108, 1993.

[2] J. C. Engwerda, A. C. M. Ran, and A. L. Rijkeboer, "Necessary and sufficient conditions for the existence of a positive definite solution of the matrix equation $X+A^{*} X^{-1} A=Q$," Linear Algebra and Its Applications, vol. 186, pp. 255-275, 1993.

[3] A. Ferrante and B. C. Levy, "Hermitian solutions of the equation $X=Q+N X^{-1} N^{*}$, , Linear Algebra and Its Applications, vol. 247, pp. 359-373, 1996.

[4] X. F. Duan, A. P. Liao, and B. Tang, "On the nonlinear matrix equation $X-\sum_{i=1}^{m} A_{i}^{*} X^{\delta_{i}} A_{i}=Q$," Linear Algebra and Its Applications, vol. 429, no. 1, pp. 110-121, 2008.

[5] X. F. Duan and A. P. Liao, "On Hermitian positive definite solution of the matrix equation $X-\sum_{i=1}^{m} A_{i}^{*} X^{r} A_{i}=Q$," Journal of Computational and Applied Mathematics, vol. 229, pp. 27-36, 2009.
[6] S. M. El-Sayed and A. M. Al-Dbiban, "On positive definite solutions of the nonlinear matrix equation $X+A^{*} X^{-n} A=I$," Applied Mathematics and Computation, vol. 151, no. 2, pp. 533541, 2004.

[7] D. J. Gao and Y. H. Zhang, "On the Hermitian positive definite solutions of the matrix equation $X-A^{*} X^{q} A=Q(q>0)$," Mathematica Numerica Sinica, vol. 29, no. 1, pp. 73-80, 2007 (Chinese).

[8] V. I. Hasanov, "Positive definite solutions of the matrix equations $X \pm A^{*} X^{-q} A=Q$," Linear Algebra and Its Applications, vol. 404, pp. 166-182, 2005.

[9] Y. Lim, "Solving the nonlinear matrix equation $X=Q+$ $\sum_{i=1}^{m} M_{i} X^{\delta i} M_{i}^{*}$ via a contraction principle," Linear Algebra and Its Applications, vol. 430, pp. 1380-1383, 2009.

[10] Y. H. Zhang, "On Hermitian positive definite solutions of matrix equation $X-A^{*} X^{-2} A=I$," Journal of Computational Mathematics, vol. 23, no. 4, pp. 408-418, 2005.

[11] L. A. Sakhnovich, Interpolation Theory and Its Applications, Mathematics and Its Applications, vol. 428, Kluwer Academic Publishers, Dordrecht, The Netherlands, 1997.

[12] A. C. M. Ran and M. C. B. Reurings, "A nonlinear matrix equation connected to interpolation theory," Linear Algebra and its Applications, vol. 379, pp. 289-302, 2004.

[13] J. G. Sun, "Perturbation analysis of the matrix equation $X=$ $\mathrm{Q}+A^{H}(\widehat{X}-C)^{-1} A$, Linear Algebra and Its Applications, vol. 372, pp. 33-51, 2003.

[14] D. J. Gao, "Existence and uniqueness of the positive definite solution for the matrix equation $X=Q+A^{*}(\widehat{X}-C)^{-1}$," Abstract and Applied Analysis, vol. 2013, Article ID 216035, 4 pages, 2013.

[15] S. M. El-Sayed and A. C. M. Ran, "On an iteration method for solving a class of nonlinear matrix equations," SIAM Journal on Matrix Analysis and Applications, vol. 23, no. 3, pp. 632-645, 2001. 
[16] A. C. M. Ran and M. C. B. Reurings, "On the nonlinear matrix equation $X+A^{*} F(X) A=Q$ : solutions and perturbation theory," Linear Algebra and Its Applications, vol. 346, pp. 15-26, 2002.

[17] A. C. Ran and M. C. B. Reurings, "A fixed point theorem in partially ordered sets and some applications to matrix equations," Proceedings of the American Mathematical Society, vol. 132, no. 5, pp. 1435-1443, 2004.

[18] A. C. M. Ran, M. C. B. Reurings, and L. Rodman, "A perturbation analysis for nonlinear selfadjoint operator equations," SIAM Journal on Matrix Analysis and Applications, vol. 28, no. 1, pp. 89-104, 2006.

[19] M. C. B. Reurings, "Contractive maps on normed linear spaces and their applications to nonlinear matrix equations," Linear Algebra and its Applications, vol. 418, no. 1, pp. 292-311, 2006.

[20] D. J. Guo, Nonlinear Functional Analysis, Shandong Science and Technology Press, Jinan, China, 2001 (Chinese).

[21] D. J. Guo and V. Lakshmikantham, Nonlinear Problems in Abstract Cones, Academic Press, London, UK, 1988.

[22] R. Bhatia, Matrix Analysis, vol. 169 of Graduate Texts in Mathematics, Springer, 1997. 


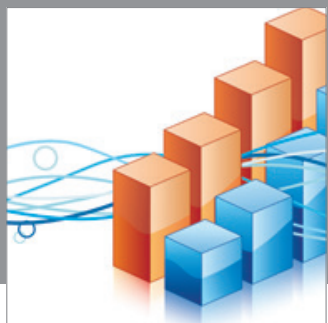

Advances in

Operations Research

mansans

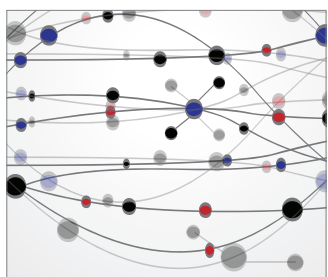

The Scientific World Journal
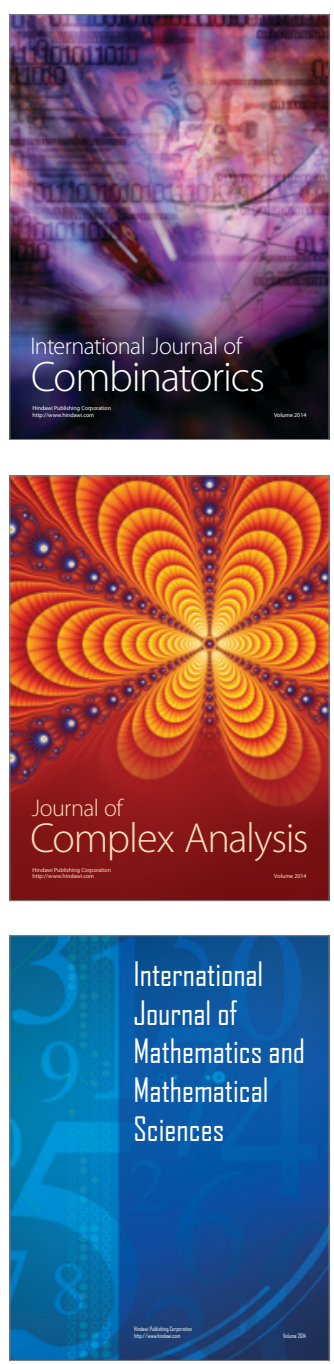
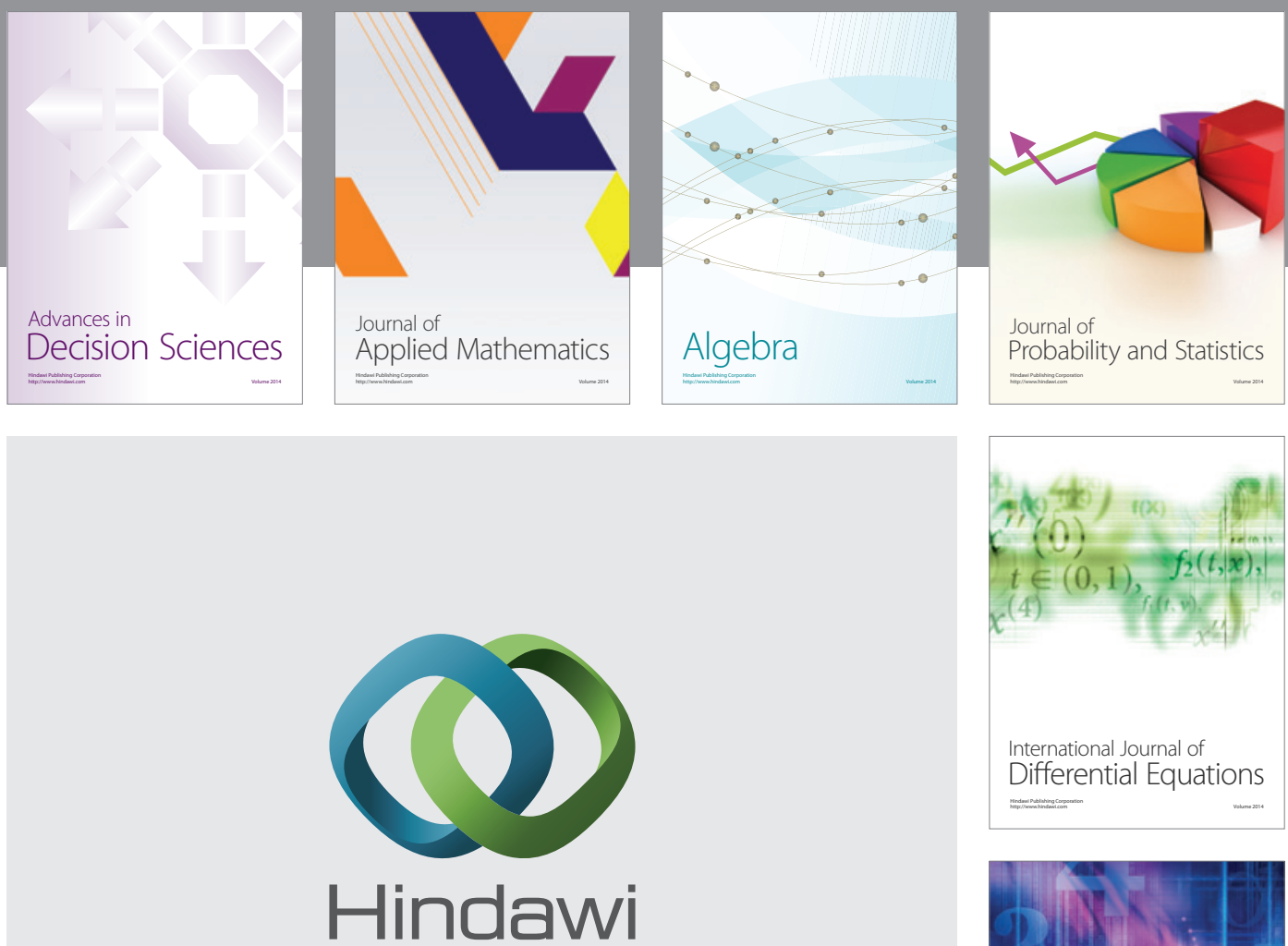

Submit your manuscripts at http://www.hindawi.com
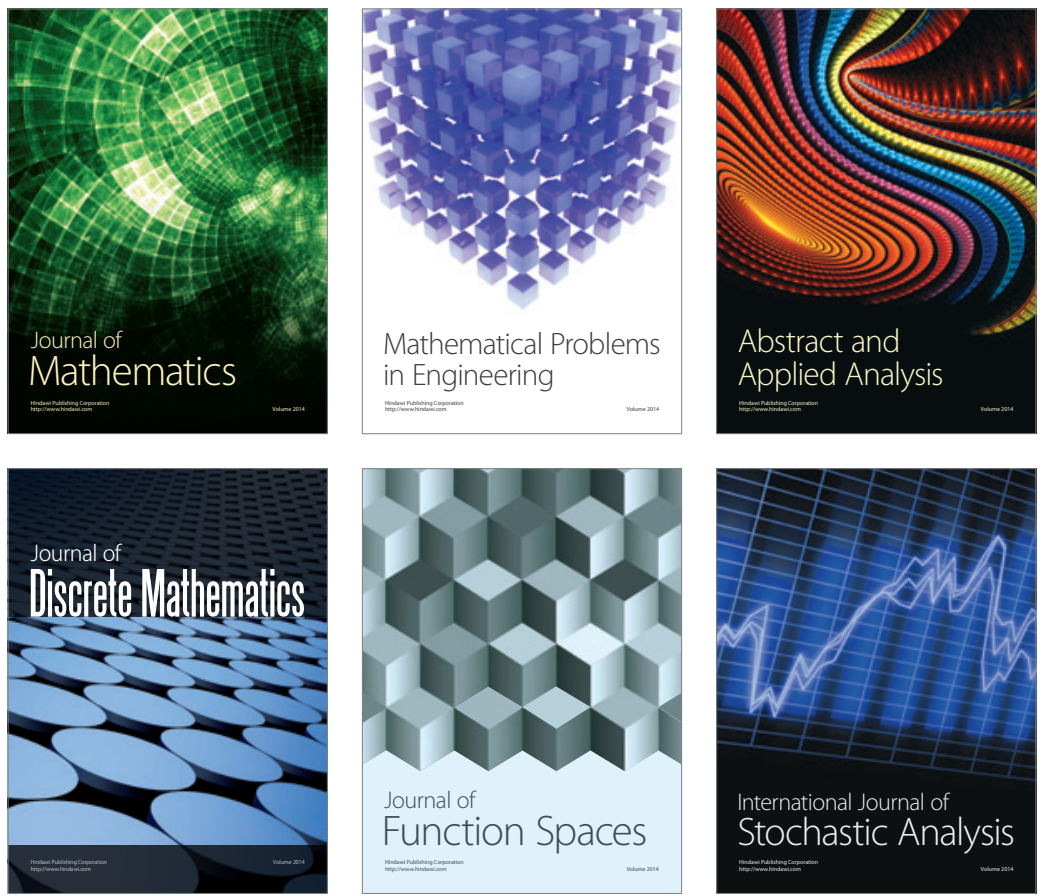

Journal of

Function Spaces

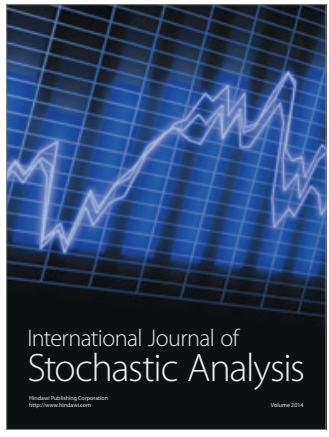

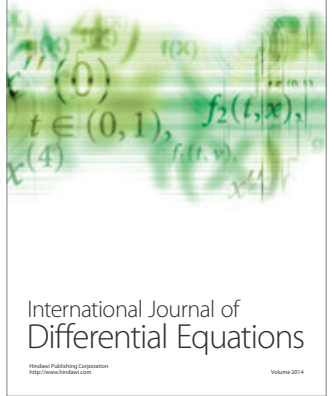
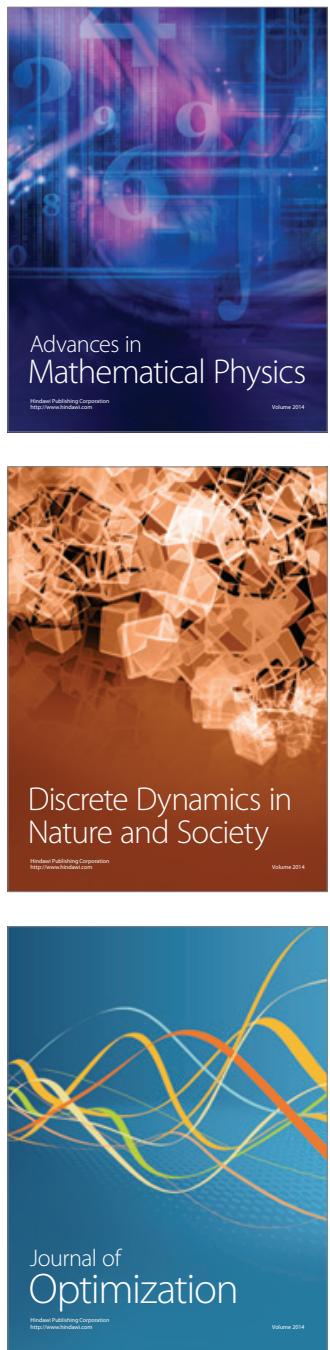